\title{
Reconstruction of shale image based on Wasserstein Generative Adversarial Networks with gradient penalty
}

\author{
Wenshu Zha, Xingbao $\mathrm{Li}^{\circledR} *$, Yan Xing, Lei He, Daolun Li \\ Department of Mathematics, Hefei University of Technology, Hefei 230009, P. R. China
}

\begin{abstract}
Keywords:
Digital core

image generation

Generative Adversarial Networks

convolutional neural network

shale

Cited as:

Zha, W., Li, X., Xing, Y., He, L., Li, D. Reconstruction of shale image based on Wasserstein Generative Adversarial Networks with gradient penalty. Advances in Geo-Energy Research, 2020, 4(1): 107-114, doi: 10.26804/ager.2020.01.10.
\end{abstract}

\begin{abstract}
:
Generative Adversarial Networks (GANs), as most popular artificial intelligence models in the current image generation field, have excellent image generation capabilities. Based on Wasserstein GANs with gradient penalty, this paper proposes a novel digital core reconstruction method. First, a convolutional neural network is used as a generative network to learn the distribution of real shale samples, and then a convolutional neural network is constructed as a discriminative network to distinguish reconstructed shale samples from real ones. Through this confrontation training method, realistic digital core samples of shale can be reconstructed. The paper uses two-point covariance function, Fréchet Inception Distance and Kernel Inception Distance, to evaluate the quality of digital core samples of shale reconstructed by GANs. The results show that the covariance function can test the similarity between generated and real shale samples, and that GANs can efficiently reconstruct digital core samples of shale with high-quality. Compared with multiple point statistics, the new method does not require prior inference of the probability distribution of the training data, and directly uses noise vector to generate digital core samples of shale without using constraints of "hard data" in advance. It is easy to produce an unlimited number of new samples. Furthermore, the training time is also shorter, only 4 hours in this paper. Therefore, the new method has some good points compared with current methods.
\end{abstract}

\section{Introduction}

As a major component of natural gas supply, shale contains a multi-scale pore structure, ranging from micrometer to nanometer scale. Its pore size directly affects the flow mechanism of shale gas such as adsorption, diffusion and slippage (Li et al., 2016). Because it is expensive to obtain the high-resolution shale digital rock, researchers usually use the reconstructed pore structure to evaluate shale gas-bearing properties (Zhang et al., 2017).

There are three kinds of methods for reconstructing digital cores: physical experiment methods, numerical reconstruction methods, and hybrid modeling methods. In physical experiment methods, various imaging tools are used to reconstruct the digital core, and these tools include scanning electron microscope (SEM) (Scott et al., 2019), focused ion beam scanning electron microscope (FIB-SEM) (Liu et al., 2017), and Nano-CT (Zuluaga et al., 2014). SEM is mainly used to capture a large number of two-dimensional images. The limitation of this method is that only two-dimensional surfaces of the sample can be observed at a time. FIB-SEM grinds the surface of the sample. During the grinding process, the physical form of the sample may be lost, resulting in impaired observation accuracy. Nano-CT scan (Lin et al., 2015) is the most widely used method, which has the advantage of high accuracy, but the disadvantage is that there is a trade-off between the volume and resolution of the imaging.

Different from physical experiment methods, the numerical reconstruction methods use digital microscope scanning or Nano-CT scanning pictures to perform digital core reconstruction. The common methods include the process-based method (PBM) (Yao et al., 2005; Ji et al., 2018), simulated annealing method (SAM) (Jiao et al., 2008), Markov chain Monte Carlo method (MCMCM) (Zhang et al., 2014), truncated Gaussian random field method (TGRFM) (Lin et al., 2018), multiple point statistics (MPS) (Okabe and Blunt, 2007; Zhang et al., 2010; Tahmasebi et al., 2012), superposition coupling algorithm (Yang et al., 2015).

*Corresponding author.

E-mail address: wszha@hfut.edu.cn (W. Zha); 15212445868@163.com (X. Li); xy1128@126.com (Y. Xing);

hlei80@163.com (L. He); ldaol@ustc.edu.cn (D. Li).

2207-9963 (c) The Author(s) 2020.

Received February 16, 2020; revised March 5, 2020; accepted March 5, 2020; available online March 9, 2020. 
The advantage of PBM is that it can reconstruct a more ideal pore structure. The disadvantage is that the method is complicated, the calculation cost is high, and the implementation is difficult. MPS mainly uses training samples instead of variograms to represent the spatial structure of geological variables, thereby randomly reconstructing images similar to the training images. Its advantage is that it uses less CPU and memory load during the reconstruction of rock samples. The disadvantage is that the structural characteristics of the reconstructed core in the vertical directions are similar to horizontal directions. It is possible to obtain isotropic reconstruction results, and it cannot reflect the characteristics of the true pore structure. In addition, "hard data" need to be given in advance when reconstructing digital cores, and is the result of measuring and observing something or phenomenon that exists objectively. To overcome the limitation of a single method, Lin et al. (2017) proposed a hybrid modeling method combining numerical reconstruction and physical experiment. This method retains important microstructure information of microfracture images. In general, hybrid methods not only improve the accuracy and efficiency of modeling to a certain extent, but also overcome many limitations of separate modeling method.

With the development of high-performance computing and deep learning, learning reproducible feature representations from the original unlabeled data set has attracted wide attention in computer vision (Radford et al., 2015). In recent years, the most popular unsupervised models for learning feature representations are Generative Adversarial Networks (GANs), which use unique adversarial training ideas to generate highquality samples. Currently, GANs have achieved remarkable success in the field of image generation. Progressive Growing of GANs (PG-GANs) (Karras et al., 2017), as a major breakthrough in the development history of GANs, achieved a leapforward improvement in the quality of image generation and successfully generated realistic high-definition images with a resolution $1024 \times 1024$. The fingerprints generated by GANs pose a certain threat to the security of fingerprint unlocking. They are used to attack the fingerprint unlocking of mobile phones, and the cracking success rate is up to $78 \%$ (Bontrager et al., 2017). It can be seen that GANs have excellent image generation capabilities.

To this end, this paper proposes a new method to reconstruct digital core of shale based on deep learning. The new model directly learns from real shale images and captures the feature statistics of the original image set without needing any manual feature extraction. Digital core images can be reconstructed by sampling and decoding noise vectors in the latent space.

\section{Generative Adversarial Networks}

GANs (Goodfellow et al., 2014) are a class of unsupervised methods that directly learn the probability distribution of data without generating prior inferences about the probability distribution related with training data, and then use them to generate samples. GANs are mainly composed of a generative network and a discriminative network. The generative network takes noise vectors as input and generates samples similar to real samples, i.e., generated ones. The discriminative network is mainly used to distinguish whether the input samples are from real or generated samples. In the confrontation between the two, they have continuously improved their respective generation and discrimination capabilities. Although GANs can generate attractive samples, it is difficult to train due to fact that GANs are prone to mode collapse or irregular textures problems in the generated samples.

In recent years, various variants of GANs have appeared, such as Deep Convolutional GANs (DCGANs) (Radford et al., 2015), Least Square GANs (LSGANs) (Mao et al., 2017), Wasserstein GANs (WGANs) (Arjovsky et al., 2017). They try to make GANs train stably and generate high-quality samples. But these GANs variants still suffer from instability during training. As one of the important variants of GANs, Wasserstein GANs with gradient penalty (WGAN-GP) follows the convolutional architecture of DCGANs. Their main difference is the loss function. DCGANs uses a Cross Entropy loss function, while WGAN-GP uses a Wasserstein loss function. Based on the latter's convolution properties and improvement of the loss function, WGAN-GP (Gulrajani et al., 2017) greatly improves the stability of training and the quality of generated samples.

In the process of constructing the WGAN-GP model, shale image $x$ is defined as a sample that obeys a real probability density function $P_{r}$. Two differentiable functions with parameters $\theta$ and $\varphi$ are used to represent the generative network $G_{\theta}(z)$ and discriminative network $D_{\varphi}(x)$, and backpropagation and batch gradient descent are used to optimize generative network $G$ and discriminative network $D$ according to the function:

$V_{W G A N-G P}\left(D_{\varphi}, G_{\theta}\right)=\max _{D_{\varphi}}\left\{\begin{array}{l}E_{x \sim p_{r}}\left[D_{\varphi}(x)\right]-E_{x \sim p_{g}}\left[D_{\varphi}(x)\right] \\ -\lambda E_{x \sim p_{m}}\left(\left\|\nabla_{x} D_{\varphi}(x)\right\|-1\right)^{2}\end{array}\right\}$

where $P_{r}$ in Eq. (1) represents the distribution of real samples, $P_{g}$ in Eq. (1) represents the distribution of generated samples, $P_{m}$ in Eq. (1) is implicitly defined along straight lines between pairs of points sampled from $P_{r}$ and $P_{g}$.

The training of WGAN-GP is mainly performed in two steps: the first step is to train the discriminative network to maximize its ability to distinguish between real and fake shale samples, and the second step is to train the generative network to maximize its ability to deceive the discriminative network, so that the shale samples generated by the generative network are misclassified as real ones. WGAN-GP will suffer from training instability during training, and it takes a lot of experiments to find the best set of parameters for stable training.

\section{Digital core image reconstruction method based on Generative Adversarial Networks}

\subsection{GANs model training flowchart}

Fig. 1 shows the training flow of the GANs model. As shown in figure, the generative network takes the noise 


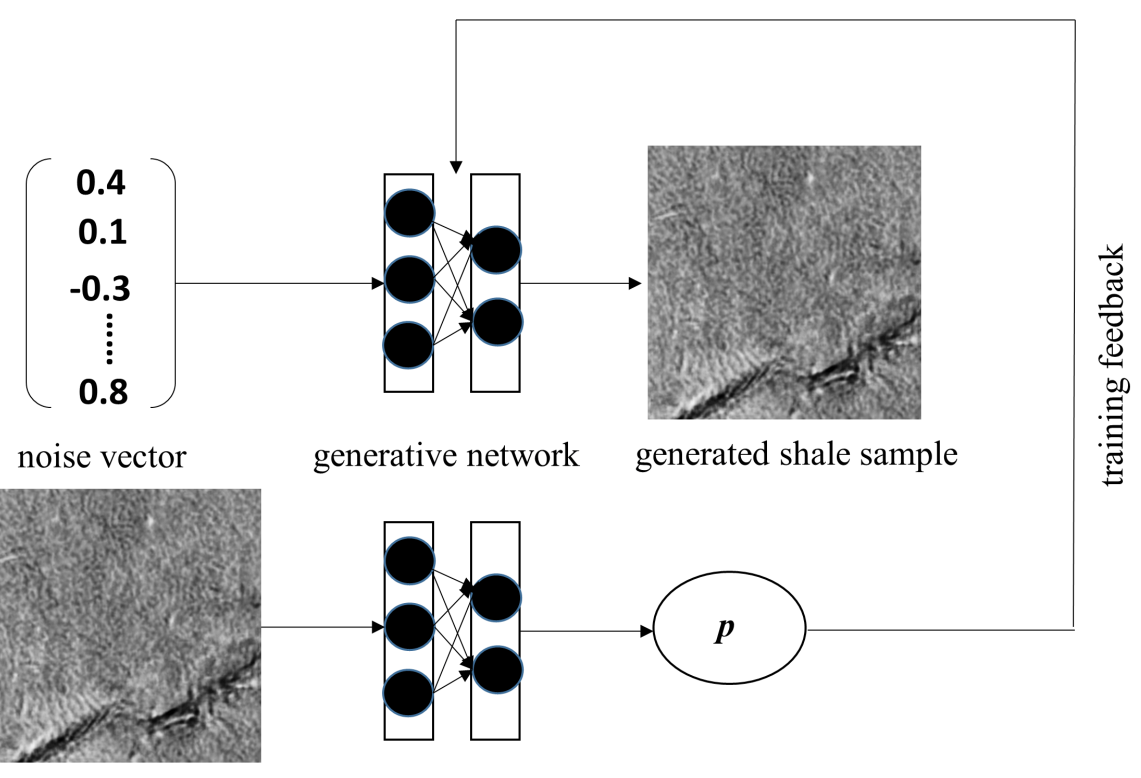

real and generated shale sample discriminative network

Fig. 1. The training flowchart of GANs model.

vector as input, and its output is the generated shale sample. The discriminative network takes the real shale sample and the generated one as input, and its output is a scalar value $P$, which represents the probability that the input sample is from the real shale sample rather than generated one. Through training feedback, the generative network and the discriminative network compete with each other in the training process, and continuously improve their own generating and discriminating capabilities, respectively, until the discriminative network cannot judge whether the input sample is from a real shale sample or a generated one.

\subsection{WGAN-GP algorithm}

WGAN-GP, like GANs, is also composed of a generative network and a discriminative network. The generative network is mainly responsible for generating samples with a probability density of $P_{r}$. The discriminative network is mainly used to distinguish whether the input samples are from real or generated shale samples. The generative network $G$ defined by its parameters $\theta$ is a mapping from the prior noise $z$ of the latent space to the shale image domain:

$$
\begin{gathered}
z \sim N(0,1)^{n \times d} \\
G_{\theta}: z \sim R^{256 \times 256}
\end{gathered}
$$

where $N(0,1)$ in Eq. (2) represents a Gaussian distribution with a mean of 0 and a standard deviation of $1, n \times d$ in Eq. (2) is the dimension of the prior noise, and $256 \times 256$ in Eq. (3) is the size of shale samples.
The discriminative network $D$ defined by its parameters $\varphi$ is a function that maps samples of shale image domain to intervals $[0,1]$ :

$$
D_{\varphi}: R^{256 \times 256} \sim[0,1]
$$

where interval $[0,1]$ in Eq. (4) represents the probability that the input sample is from the real shale sample rather than generated one.

Requirements: The number of discriminative network $D$ iterations $N_{D}$, the batch size $n$, the gradient penalty coefficient $\lambda$, the learning rate $\eta$. Randomly initialize the parameters of $D$ and $G$.

1): while generative network $G$ has not converged do;

2): for $k=1,2, \ldots, N_{D}$ do;

3): Sample $n$ real shale samples $x^{(i)}$ from $P_{r}(x)$, sample $n$ noise vector $z^{(i)}$ from $N(0,1)^{n \times d}$, thereby obtaining $n$ generated shale samples $\tilde{x}^{(i)}=G\left(z^{(i)}\right)$, sample a random weighted number $\alpha$ from the uniform distribution, thereby obtaining $n$ weighted average samples $\hat{x}^{(i)}=\alpha x^{(i)}+(1-\alpha) \tilde{x}^{(i)}$, where $i=1,2, \ldots, n$;

4): The parameters $\varphi$ of $D$ are updated by the batch gradient ascent method to maximize the following formula:

$$
\begin{gathered}
V=\frac{1}{n} \sum_{i=1}^{n}\left[D\left(x^{(i)}\right)-D\left(\tilde{x}^{(i)}\right)-\lambda\left(\left\|\nabla_{x} D\left(\hat{\wedge}^{(i)}\right)\right\|-1\right)^{2}\right], \\
\varphi \leftarrow \varphi+\eta \nabla V(\varphi)
\end{gathered}
$$

5): end for;

6): Sample $n$ noise vector $z^{(i)}$ from $N(0,1)^{n \times d}$, thereby obtaining $n$ generated shale samples $\tilde{x}^{(i)}=G\left(z^{(i)}\right)$; 
7): The parameters $\theta$ of $G$ are updated by the batch gradient descent method to minimize the following formula:

$$
\tilde{V}=-\frac{1}{n} \sum_{i=1}^{n} D\left(\tilde{x}^{(i)}\right), \quad \theta \leftarrow \theta-\eta \nabla \tilde{V}(\theta)
$$

8): end while.

\section{Model evaluation method}

Evaluating the performance of GANs models is inherently challenging. This paper selects two-point covariance function and the similarity indexes of measuring difference of GANsgenerated and real shale samples, namely Fréchet Inception Distance (FID) (Heusel et al., 2017) and Kernel Inception Distance (KID) (Bińkowski et al., 2018) to evaluate the final WGAN-GP model.

\subsection{Two-point covariance function}

The second-order moment of the microstructure can be characterized by a two-point probability function (Mosser et al., 2017). By the assumption of stationarity, it is equivalent to the two-point covariance function $S_{2}(r)$ :

$$
S_{2}(r)=\boldsymbol{P}(x \in P, x+r \in P), \quad \text { for } x, r \in R^{h}
$$

Arbitrary two points separated by the separation lag distance $r$ are located in the same phase, which is the void phase or grain phase of the microstructure, although $S_{2}(r)$ can be defined for the two phases of a porous medium, the twopoint covariance function relative to the pore phase $P$ only is computed. Eq. (7) represents the probability that two points $x$ and $x+r$ separated by the separation distance $r$ are located in the pore phase $P$. To estimate the two-point covariance function, the Ostu thresholding method (Ostu, 1979) is used to segment all images. For the WGAN-GP model, we use the training image and the generated image of WGAN-GP model to estimate the two-point covariance function $S_{2}(r)$.

\subsection{Fréchet Inception Distance and Kernel Inception Distance}

Heusel et al. (2017) proposed the FID to calculate the distance between the real samples and the generated ones in the feature space to evaluate the model's ability to generate real samples. For feature functions $\varphi$ (by default, the convolutional features of the Inception network), $\varphi\left(P_{r}\right)$ and $\varphi\left(P_{g}\right)$ are modeled as Gaussian random variables with empirical means $\mu_{r}, \mu_{g}$ and empirical covariance $\Sigma_{r}, \Sigma_{g}$. The Fréchet distance between $P_{r}$ and $P_{g}$ can be calculated using the following formula,

$$
F I D\left(P_{r}, P_{g}\right)=\left\|\mu_{r}-\mu_{g}\right\|+T_{r}\left(\Sigma_{r}+\Sigma_{g}-2\left(\Sigma_{r} \Sigma_{g}\right)^{1 / 2}\right)
$$

where $P_{r}, P_{g}$ in Eq. (8) are probability distributions obeyed by real samples and generated ones, respectively; $T_{r}$ in Eq. (8) is the trace of covariance matrix. A lower FID value means that the Gaussian distribution distance between the real samples and the generated ones is smaller, and the quality of the generated ones is higher.

Bińkowski et al. (2018) proposed the KID to evaluate the model's ability to generate real samples, which is the square of the Maximum Mean Discrepancy (MMD) between two Inception representations. The Kernel Inception Distance between and can be computed using the following formula:

$$
\begin{aligned}
K I D\left(P_{r}, P_{g}\right)= & E_{x, \hat{x} \sim P_{r}}[k(x, \hat{x})]+E_{y, \hat{y} \sim P_{g}}[k(y, \hat{y})] \\
& -2 E_{x \sim P_{r}, y \sim P_{g}}[k(x, y)]
\end{aligned}
$$

where $P_{r}, P_{g}$ in Eq. (9) are probability distributions obeyed by real samples and generated ones, respectively; a polynomial kernel is represented by $k(x, y)=\left((1 / d) x^{\mathrm{T}} y+1\right)^{3}, d$ is the dimension, and the purpose of using a polynomial kernel is to avoid adjusting any kernel parameters (Pedregosa et al., 2011). Eq. (9) measures the difference in the distribution of visual features between real samples and generated ones. The lower the KID value, the smaller the Gaussian distribution distance between the generated samples and the real ones. In other words, the quality of the generated samples is higher. Different from FID, to make the estimation between comparison models more intuitive, the formula calculates asymptotic and unbiased normal estimates.

\section{Experiment and result analysis}

\subsection{Shale datasets}

Nano-CT technology has been widely used in shale reservoir research (Tahmasebi et al., 2015). In this experiment, the sample comes from Wufeng, Jiaoshiba Block. It was collected by Nano-CT technology, and cracks and white ores are main characteristics it contains. Fig. 2 shows a slice of the shale sample CT image with a resolution of $60 \mathrm{~nm} / \mathrm{pixel}$. The original shale image is randomly cropped into 10,000 training samples of size $256 \times 256$. Before using the original image as a training samples, except for random cropping, there is no other preprocessing such as denoising, threshold segmentation.

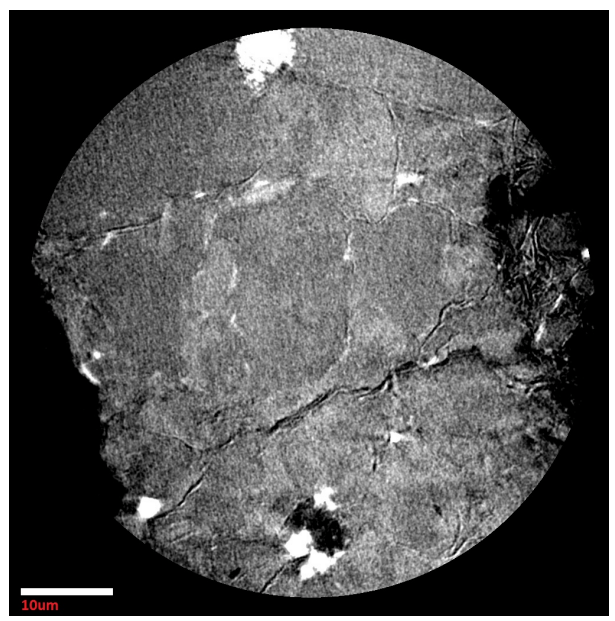

Fig. 2. Two-dimensional gray-scale cross-section of a Nano-CT image of a raw shale sample from an experimental study. 
Table 1. Main architecture configuration of WGAN-GP.

\begin{tabular}{llllll}
\hline & Layer & Filter & Stride & Batch normalization & Activation function \\
\hline Generative network & Conv2D & $(3,3,128,128)$ & 2 & yes & relu \\
& Conv2D & $(3,3,128,64)$ & 2 & yes & relu \\
& Conv2D & $(3,3,64,1)$ & 2 & no & tanh \\
\multirow{5}{*}{ Ddiscriminative network } & Conv2D & $(5,5,1,64)$ & 2 & no & leakyrelu \\
& Conv2D & $(5,5,64,128)$ & 2 & yes & leakyrelu \\
& Conv2D & $(5,5,128,256)$ & 2 & yes & leakyrelu \\
\hline
\end{tabular}

\subsection{Experimental parameters}

The training samples used in this study are 10,000 shale images with a resolution of $60 \mathrm{~nm} / \mathrm{pixel}$ and a size of 256 $\times$ 256. A three-layer convolution is used on the generative network, the Tanh activation function is applied to the output layer, all other layers use the Rectified Linear Unit (ReLU) activation function. Another three-layer convolution is used on the discriminative network, and all layers of the discriminative network use the LeakyReLU activation function, and after the convolutional layer, a Dropout layer is added; batch normalization is added in both convolutional layers of the generative network and the discriminative network. The model uses a small batch gradient descent method to network training on a single NVIDIA 1080TI GPU, the optimizer used by the model is the RMSProp optimizer. Because only three layers of convolution are used in the two networks respectively, and the Dropout layer is added to the discriminative network, the network architecture is simplified, and the network parameters are greatly reduced. Therefore the network training speed is greatly accelerated. The total time spent on training only takes about 4 hours. Table 1 shows the architecture configuration of WGAN-GP.

\subsection{Experimental results}

\subsubsection{Generation method of digital core images of shale}

GANs have a strong stochastic reconstruction capability for gray-scale shale samples, and new shale samples can be reconstructed using noise. During the generation of new shale, only the noise vector is needed. However,when reconstructing digital cores using MPS, constraints of "hard data" need to be given in advance. Compared with the MPS, GANs can generate new digital cores without any human intervention. Therefore, they are simpler and faster.

This experiment uses 10,000 shale samples of size 256 $\times 256$ as training ones, and generated shale samples are the same size as the training ones. Fig. 3 shows the original training samples and reconstructed ones based on the WGANGP model. It shows that WGAN-GP successfully learned the probability distribution of real shale samples: the shape of the generated fractures are similar to that of real fractures.

\subsubsection{Diversity of digital core images of shale}

As shown in Fig. 3, the real and generated shale have similar characteristic of fractures and white ores. Compared with the real shale samples, the generated ones have different structural morphology of the fractures, which can be seen from the second column of shale samples marked by red boxes. It can also be observed from the third column of generated samples marked by red oval frames that the structural morphology and location of the white ores have changed to varying degrees. The reason for these phenomena is that GANs successfully learned the fractures and white ores characteristics of real shale samples, so these characteristics will be reproduced in the generated ones.

\subsubsection{Structure consistency of digital core images of shale}

As shown in Fig. 4, the two-point covariance functions
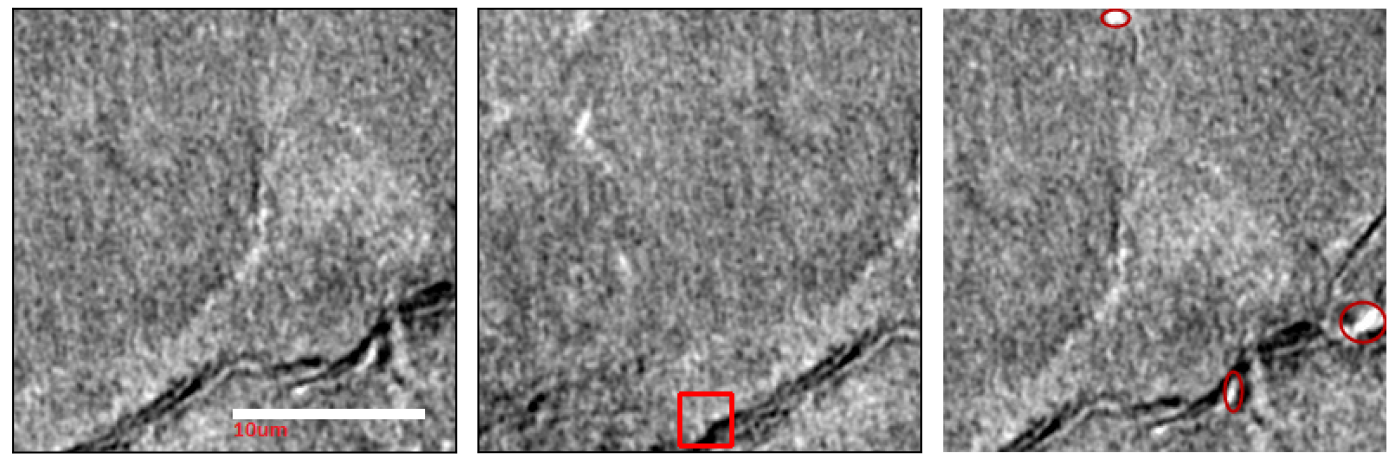

(a) Real shale samples 

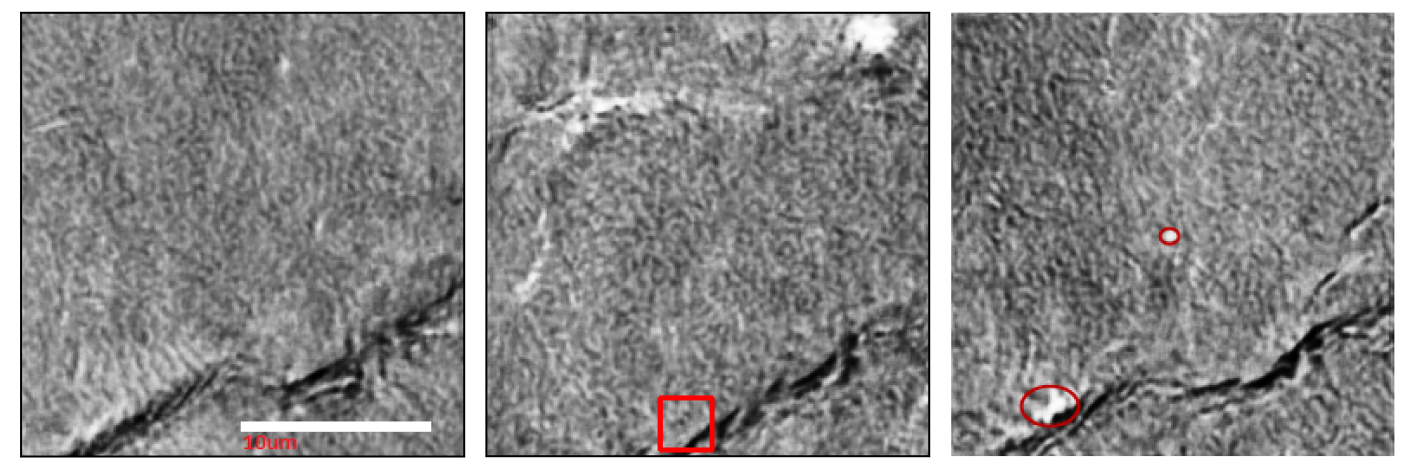

(b) Generated shale samples 1
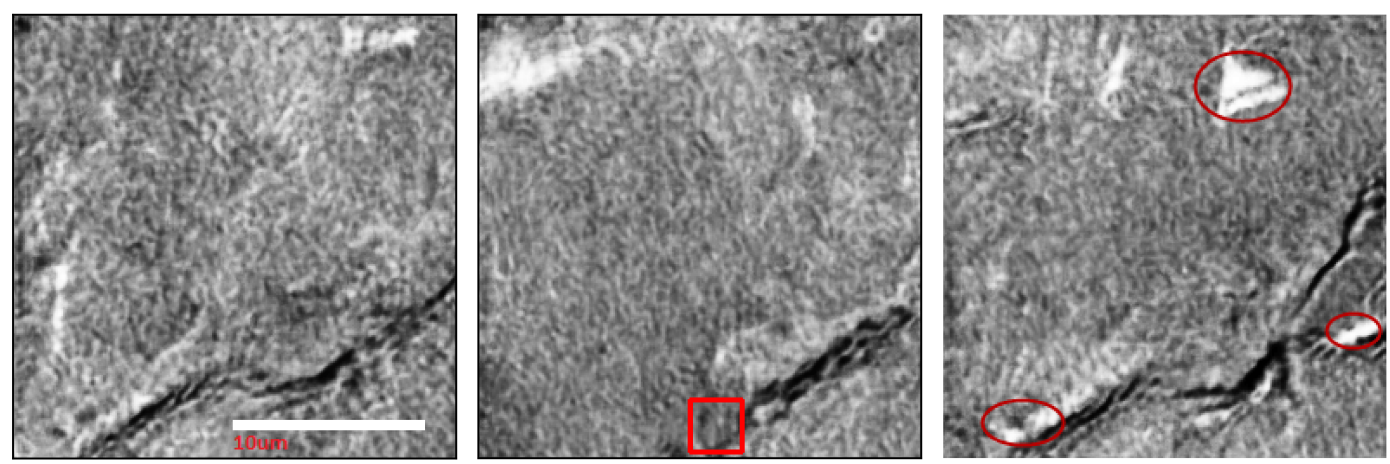

(c) Generated shale samples2

Fig. 3. Comparison between real shales and generated ones of WGAN-GP model.

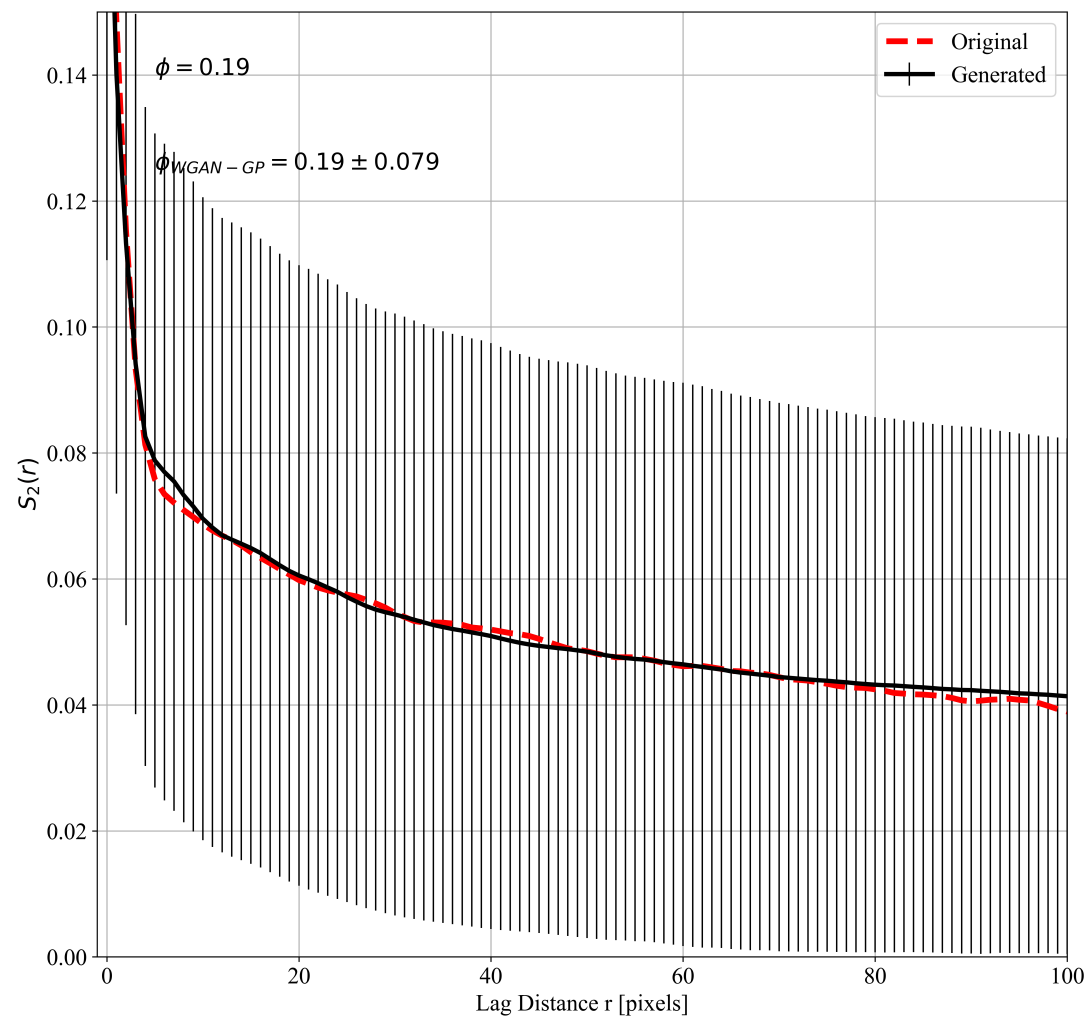

Fig. 4. Comparison of radial average covariance functions of real shale samples and 100 generated shale samples of WGAN-GP model. 
Table 2. FID and KID values of real shale samples and generated shale samples.

\begin{tabular}{lll}
\hline Models & FID Score & KID Score \\
\hline Real samples & 16.63 & $5.11 \pm 0.19$ \\
WGAN-GP & 91.12 & $6.38 \pm 0.57$ \\
DCGAN & 136.51 & $11.73 \pm 0.84$ \\
\hline
\end{tabular}

$S_{2}(r)$ show an approximate exponential decay state for both real shale samples and generated ones. The obtained covariance functions are stable at almost all pixels. The covariance function measured on the generated shale samples shows excellent agreement with the real ones, indicating that generated and real shale samples are very close on the fracture structure. Where $\phi$ is the ratio of organic matter components in real shale samples, and 0.079 is the standard deviation of the ratio of organic matter components in generated ones.

\subsubsection{Gaussian consistency of digital core images of shale}

In the experiment, the FID and KID algorithms are used to compute FID and KID values on 100 real shale samples, 100 ones generated by WGAN-GP, and 100 ones generated by DCGANs. As shown in Table 2, the FID and KID values of the shale samples generated by WGAN-GP model are higher than those of the real shale samples, but lower than those of the ones generated by DCGANs model. Therefore, the quality of shale samples generated by WGAN-GP model is closer to that of real ones, which shows that generated and real shale samples have better consistency in the Gaussian distribution they obey.

\section{Discussion}

Although GANs can generate visually attractive highquality samples, GANs also have some potential drawbacks. For example, GANs are very sensitive to parameters. Even if the parameters change slightly, the gradient may disappear easily, and the model would not converge. Although WGAN-GP alleviates the phenomenon, it does not completely eliminate it, so it takes a lot of time to adjust the parameters. Moreover, similar to other neural networks, GANs also have a black box mechanism. As a result, it is very challenging to understand that the internal mechanism of neural networks that reconstruct high-quality samples, i.e., the lack of interpretability, which is difficult to solve. In addition, the samples generated by GANs are random, so the size and position distribution of the characteristics in the generated samples cannot be accurately controlled.

\section{Conclusions}

This paper proposed a new digital core image reconstruction method based on GANs. The main conclusions are as follows.

1) GANs can effectively reconstruct digital core images.
They use shale samples for unsupervised training, and do not need to manually extract characteristics. They converge quickly, and the generated samples are of high quality and rich in diversity.

2) GANs can generate digital core images with high quality. The generated digital core images have good consistency with real ones. Generated digital core images can effectively capture fractures and white ores characteristics. The indicators of FID and KID further illustrate the consistency of the Gaussian distribution that generated and real digital core images obey, and quantitatively show that they have good consistency in image quality.

3) Research on digital core images reconstruction based on GANs has an important application value. The GANs kernel used in this paper is a convolutional neural network with powerful image processing capabilities. The characteristics of generated digital core images are consistent with those of real digital core images. In addition, digital cores can be produced directly without the need for "hard data" constraints. GANs have the most fusion ability, and is expected to generate richer and more diverse digital core samples. This has great significance for digital core research.

\section{Acknowledgement}

This work was supported by National Science and Technology Major (No: 2017ZX05009005-002).

\section{Conflict of interest}

The authors declare no competing interest.

Open Access This article, published at Ausasia Science and Technology Press on behalf of the Division of Porous Flow, Hubei Province Society of Rock Mechanics and Engineering, is distributed under the terms and conditions of the Creative Commons Attribution (CC BY-NC-ND) license, which permits unrestricted use, distribution, and reproduction in any medium, provided the original work is properly cited.

\section{References}

Arjovsk, M., Chintala, S., Bottou, L. Wasserstein GAN. Paper Presented at the International Conference on Machine Learning, Sydney, Australia, 6-11 August, 2017.

Bińkowski, M., Sutherland, D.J., Arbel, M., et al. Demystifying mmd gans. Paper Presented at the International Conference on Machine Learning, Stockholm, Sweden, 10-15 July, 2018.

Bontrager, P., Roy, A., Togelius, J., et al. Deepmasterprints: Generating masterprints for dictionary attacks via latent variable evolution. Paper Presented at the International Conference on BTAS, Los Angeles, USA, 22-25 October, 2018.

Goodfellow, I., Pouget-Abadie, J., Mirza, M., et al. Generative adversarial nets. Paper Presented at the International Conference on Advances in Neural Information Processing Systems, Montreal, Canada, 8-13 December, 2014.

Gulrajani, I., Ahmed, F., Arjovsky, M., et al. Improved training of Wasserstein GANs. Paper Presented at 
the International Conference on Advances in Neural Information Processing Systems, Long Beach, USA, 4-9 December, 2017.

Heusel, M., Ramsauer, H., Unterthiner, T., et al. Gans trained by a two time-scale update rule converge to a local nash equilibrium. Paper Presented at the International Conference on Advances in Neural Information Processing Systems, Long Beach, USA, 4-9 December, 2017.

Ji, L., Lin, M., Jiang, W., et al. An improved method for reconstructing the digital core model of heterogeneous porous media. Transp. Porous Media 2018, 121(2): 389406.

Jiao, Y., Stillinger, F.H., Torquato, S. Modeling heterogeneous materials via two-point correlation functions: II. Algorithmic details and applications. Phys. Rev. E 2008, 77(3): 031135.

Karras, T., Aila, T., Laine, S., et al. Progressive growing of GANs for improved quality, stability, and variation. Paper Presented at the International Conference on Learning Representations, Toulon, France, 24-26 April, 2017.

Li, D., Zhang, L., Wang, J., et al. Composition-Transient analysis in shale-gas reservoirs with consideration of multicomponent adsorption. SPE J. 2016, 21(2): 648-664.

Lin, C., Wu, Y., Ren, L., et al. Review of digital core modeling methods. Progress in Geophysics 2018, 33(2): 679-689. (in Chinese)

Lin, M., Jiang, W., Li, Y., et al. Several questions in the micro-scale flow of shale oil/gas. Bulletin of Mineralogy, Petrology and Geochemistry 2015, 34(1): 18-28. (in Chinese)

Lin, W., Li, X., Yang, Z., et al. Construction of dual pore 3-D digital cores with a hybrid method combined with physical experiment method and numerical reconstruction method. Transp. Porous Media 2017, 120(1): 227-238.

Liu, S., Sang, S., Wang, G., et al. FIB-SEM and X-ray CT characterization of interconnected pores in high-rank coal formed from regional metamorphism. J. Petrol. Sci. Eng. 2017, 148: 21-31.

Mao, X., Li, Q., Xie, H., et al. Least squares generative adversarial networks. Paper Presented at the International Conference on Computer Vision and Pattern Recognition, Hawaii, USA, 21-26 July, 2017.

Mosser, L., Dubrule, O., Blunt, M.J. Reconstruction of threedimensional porous media using generative adversarial neural networks. Phys. Rev. E 2017, 96(4): 043309.

Okabe, H., Blunt, M.J. Pore space reconstruction of vuggy carbonates using microtomography and multiplepoint statistics. Water Resour. Res. 2007, 43(12): 3-7.

Otsu, N. A Threshold selection method from gray-level histograms. IEEE Trans. Syst. Man Cybern. 1979, 9(1): 62-66.

Pedregosa, F., Varoquaux, G., Gramfort, A., et al. Scikit-learn: Machine learning in Python. J. Mach. Learn. Res. 2011, 12: 2825-2830.

Radford, A., Metz, L., Chintala, S. Unsupervised representation learning with deep convolutional generative adversarial networks. Paper Presented at the International Conference on Learning Representations, San Diego, USA, 7-9 May, 2015.

Scott, G., Wu, K., Zhou, Y. Multi-scale image-based pore space characterisation and pore network generation: Case study of a north sea sandstone reservoir. Transp. Porous Media 2019, 129(3): 855-884.

Tahmasebi, P., Hezarkhani, A., Sahimi, M. Multiple-point geostatistical modeling based on the crosscorrelation functions. Comput. Geosci. 2012. 16(3): 779-797.

Tahmasebi, P., Javadpour, F., Sahimi, M. Three-dimensional stochastic characterization of shale SEM images. Transp. Porous Media 2015, 110(3): 521-531.

Yang, Y., Yao, J., Wang, C., et al. New pore space characterization method of shale matrix formation by considering organic and inorganic pores. J. Nat. Gas Sci. Eng. 2015, 27: 496-503.

Yao, J., Zhao, X., Yi, Y., et al. The current situation and prospect on digital core technology. Petroleum Geology and Recovery Efficiency 2005, 12(6): 52-54. (in Chinese)

Zhang, J., Li, S., Wang, L., et al. A new method for calculating gas saturation of low-resistivity shale gas reservoirs. Nat. Gas Ind. B 2017, 4(5): 346-353.

Zhang, L., Ning, Z., Shi, P. Input-Output approach to control for fuzzy Markov jump systems with time-varying delays and uncertain packet dropout rate. IEEE Trans. Cybernet. 2014, 45(11): 2449-2460.

Zhang, T., Li, D., Lu, D., et al. Research on the reconstruction method of porous media using multiple-point geostatistics. Sci. China Phys. Mechan. Astron. 2010, 53(1): 122-134.

Zuluaga, M.A., Orkisz, M., Dong, P., et al. Bone canalicular network segmentation in 3D Nano-CT images through geodesic voting and image tessellation. Phys. Med. Biol. 2014, 59(9): 2155-2171. 